\title{
ARC WELDING MACHINES OF THE WILSON WELDER AND METALS COMPANY
}

\author{
BY ALEXANDER CHURCHWARD
}

Engineer, Wilson Welder and Metals Co.

$\mathbf{W}^{\mathrm{H}}$

HILE experimenting with alloy wire to get greater strength than that obtained with the ordinary low-carbon wire, it was discovered that with a long arc a high-carbon content wire lost most of its carbon while going through the arc.

Having the above in view, a system was developed, whereby a long arc cannot be drawn; 16 to 19 volts is accepted as a normal arc. There must be enough stabilizing resistance used to allow the arc momentarily to be drawn out to 22 volts, to take care of inequalities, of the piece to be welded, the burning off of the electrode and unsteadiness of the operator's hand. The lowest voltage to take care of this condition with ordinary resistance grids, non-automatic, was found to be 60 , but this would give too long an arc with the ordinary welder, it gave a flexible arc to be sure, but with the ordinary welder, a flexible are is dangerous if good welds are required.

Therefore, to get a normal arc without dangerous flexibility, it was found that 35 to 37.5 volts was the maximum that could be used. Now, 35 volts with a fixed resistance will not give a steady or constant current. It had also been determined that unless the current was constant good welds of the maximum strength could not be made. It was decided to devise an automatic resistance, namely the carbon pile.

The function of the carbon pile is briefly as follows:

First: The carbon pile is in series with the arc.

Second: A heavy spring compresses the pile to minimum resistance and adjustments may be made for current values required. 
Third: A solenoid in series with the welding circuit, counteracts the spring pressure so that any predetermined current value can be maintained.

The generator is of the constant-potential low-voltage type (35 to 37.5 -volt) flat compounded. When the electrode is short-circuited on the work the voltage of the generator remains constant, and does not drop to zero, to be built up when the are is started, but the solenoid instantaneously functions, and releases the pressure on the carbon pile, thus, inserting the proper amount of resistance automatically, preventing the short-circuiting current on the surge from rising more than 10 per cent above the welding current. As the arc lengthens and requires more voltage, the carbon pile, controlled by the solenoid, instantaneously responds and the current when welding is kept constant within 5 per cent.

This type of control was selected because its time element was much less than some other types of control. It has especially proved itself in multiple arc machines, two or more welders operating from the same machine, sometimes on the same piece of work.

What is claimed for this system is that there is produced constant heat per unit area in the weld, not in the arc.

Standard machines are made in three capacities, one-,two- and four-operator machines for all available circuits, both a-c. and d-c. Also gas-driven one- and two-operator sets, and belted machines to be driven off line shafts. 